\title{
Crescimento de mudas de Eucalyptus camaldulensis em substratos à base de casca de arroz carbonizada
}

\author{
Dagma Kratz ${ }^{*}$, Ivar Wendling ${ }^{2}$ \\ 10.1590/0034-737X201663030011
}

\section{RESUMO}

Com base na importância do substrato na produção de mudas florestais, objetivou-se, com este trabalho, avaliar a viabilidade técnica de 13 substratos, preparados com misturas de substrato comercial à base de casca de pinus e vermiculita (SC), casca de arroz carbonizada com diferentes granulometrias [maior que $2 \mathrm{~mm}$ (CAC1), CAC com granulometria entre 1-2 mm (CAC2), CAC com granulometria entre 0,5 - $1 \mathrm{~mm}$ (CAC3), CAC com granulometria menor que $0,5 \mathrm{~mm}$ (CAC4)], fibra de coco (FC) e vermiculita média (VM), na produção de mudas de Eucalyptus camaldulensis, e a correlação de suas propriedades físicas e químicas com seu crescimento. Foram utilizados tubetes de $55 \mathrm{~cm}^{3}$. As mudas permaneceram 60 dias em estufa de vidro e 30 dias a pleno sol. Foram avaliadas a altura, diâmetro de colo, fitomassas secas aérea e radicial, facilidade de retirada do tubete, agregação das raízes ao substrato e relação entre altura e diâmetro de colo. Concluiu-se que o substrato formado por CAC pura, em diferentes granulometrias e em mistura com os outros componentes, é viável tecnicamente para a produção de mudas de Eucalyptus camaldulensis, embora aquele formado pela mistura de 50\% de CAC e VM tenha resultado em maior crescimento das mudas. As propriedades dos substratos, densidade aparente, $\mathrm{pH}$ e capacidade de troca catiônica são importantes na produção de mudas, pois apresentaram correlações significativas com as variáveis biométricas.

Palavras-chave: eucalipto, substratos renováveis, características físicas, características químicas.

\section{ABSTRACT}

\section{Growth of Eucalyptus camaldulensis seedlings in substrates based on carbonized rice hulls}

Based on the importance of substrates in the production of forest seedlings, this work aimed to evaluate the technical feasibility of 13 substrates in the production of Eucalyptus camaldulensis seedlings, also assessing the correlation between their physical and chemical properties with seedling growth. The substrates were prepared as a mixture of commercial substrate based on semi decomposed pine bark and vermiculite (CS), carbonized rice husk with different particle sizes (CAC1: greater than $2 \mathrm{~mm}$, CAC2: 1 - $2 \mathrm{~mm}, \mathrm{CAC} 30.5$ - $1 \mathrm{~mm}$ and CAC4: lower than $0.5 \mathrm{~mm}$ ), coconut fiber $(\mathrm{CF})$ and vermiculite $(\mathrm{VM})$. Tubes of $55 \mathrm{~cm}^{3}$ were used for seedling formation. The plants remained in a greenhouse for 60 days and 30 days in full-sun exposure. We evaluated: height, stem diameter, shoot dry mass, root dry weight, ease of removal from the tubes, aggregation of roots to the substrate and height-stem diameter relationship. It was concluded that the substrate consisting of pure CAC, in different particle sizes and mixed to the other components, is technically feasible for the production of Eucalyptus camaldulensis seedlings, while those formed by mixing $50 \%$ of CAC and VM resulted in increased growth of the seedlings. The properties of the substrates, bulk density, $\mathrm{pH}$ and cation exchange capacity, are important in the production of seedlings; they showed significant correlations with the biometric variables.

Keywords: eucalypt; renewable substrates; physical properties; chemical properties.

\footnotetext{
Submetido em 10/03/2014 e aprovado em 02/02/2016.

' Universidade Federal de Mato Grosso, Cuiabá, Mato Grosso, Brasil.dagkratz@yahoo.com.br

Embrapa Florestas, Colombo, Paraná, Brasil. ivar@cnpf.embrapa.br

* Autor para correspondência: dagkratz@yahoo.com.br
} 


\section{INTRODUÇÃO}

Segundo dados da Indústria Brasileira de Árvores (Ibá, 2014), a área plantada com Eucalyptus no Brasil vem crescendo ano a ano, graças a investimentos de empresas do segmento de papel e celulose. Entre as espécies mais cultivadas, em regiões tropicais com períodos de seca, está o Eucalyptus camaldulensis, espécie tolerante à deficiência hídrica do solo (Shimizu et al., 2007).

Para atender à demanda por mudas, faz-se necessário o fornecimento de plantas com qualidades fisiológica e genética superiores. Nesse sentido, muitos esforços têm sido realizados para melhorar a qualidade da muda e reduzir os custos de sua produção e, entre os fatores que influenciam essa qualidade está o substrato (Gonçalves et al., 2000; Hartmann et al., 2011). Os fatores intrínsecos dos substratos que afetam o crescimento das plantas são suas propriedades físico-químicas, que variam em função de sua origem, método de produção e proporções dos seus componentes (Wendling et al., 2007; Melo et al., 2014).

No mercado podem ser encontrados substratos prontos para produção de mudas e os principais produtos comercializados são à base de casca de pinus e turfa (Caldeira et al., 2011). Atualmente, existe baixa oferta de casca de pinus para fabricação de substratos, por causa da competição com o mercado de energia e da diminuição de plantios do gênero Pinus no Brasil (Ibá, 2014). Quanto à turfa, sua principal desvantagem é o impacto ambiental, por se tratar de um material natural não renovável (Caldeira et al., 2011), aliado à necessidade de análise fitossanitária, a fim de verificar a presença de pragas quarentenárias ou sem registro de ocorrência, veiculadas por substrato, antes da comercialização (Mapa, 2015).

A casca de arroz, resíduo encontrado nas regiões produtoras de arroz e que vem sendo utilizada como componente de substratos, após passar pelo processo de carbonização, pode ser combinada com outros materiais, como fibra de coco, vermiculita e casca de pinus, na formulação de substratos (Kratz et al., 2012, Silva et al., 2012). Segundo Couto et al. (2003), a baixa densidade da casca de arroz carbonizada é uma característica importante quando se deseja aumentar a porosidade total do substrato, proporcionando maior drenagem e melhor aeração do sistema radicial da muda. Por sua alta macroporosidade, faz-se necessária a sua combinação com elementos de maior microporosidade.

A alta macroporosidade da casca de arroz carbonizada pode ser modificada por fracionamento de suas partículas, visto que as características físicas de um substrato estão condicionadas ao tamanho e ao arranjo das partículas, em que altas proporções de frações maiores dotam o meio de elevado espaço de aeração, enquanto partículas menores fecham os poros, aumentando a microporosidade e diminuindo a macroporosidade (Fermino, 2003).
Com base no exposto e na importância do substrato e da utilização de materiais renováveis para sua formulação, este estudo objetivou avaliar a casca de arroz carbonizada pura, em diferentes granulometrias e em mistura com fibra de coco, casca de pinus semidecomposta e vermiculita para a produção de mudas de Eucalyptus camaldulensis $\mathrm{e}$ a correlação de suas propriedades físico-químicas com o crescimento das mudas.

\section{MATERIAL E MÉTODOS}

O experimento foi realizado em outubro de 2008, no Laboratório de Propagação de Espécies Florestais da Embrapa Florestas, localizada em Colombo, Paraná (25 19' 17" S e 4909’39" O). O clima da região, de acordo com o Sistema Internacional de Köppen, é do tipo Cfb (Clima subtropical úmido).

Foram preparados 13 substratos com misturas de substrato comercial à base de casca de pinus e vermiculita (SC), casca de arroz carbonizada, original e com diferentes granulometria [maior que $2 \mathrm{~mm}$ (CAC1), CAC com granulometria entre 1 - $2 \mathrm{~mm}$ (CAC2), CAC com granulometria entre 0,5 - $1 \mathrm{~mm}$ (CAC3), CAC com granulometria menor que 0,5 mm (CAC4)], fibra de coco com granulometria em torno de 0,25 a $2 \mathrm{~mm}$ (FC) e vermiculita média (VM), conforme as seguintes composições: S1- CAC; S2CAC1; S3-CAC2; S4-CAC3; S5-CAC4; S6-FC; S7-CAC/ VM (1/1, v:v); S8- CAC2/FC (1/1, v:v); S9- CAC3/FC (1/1, v:v); S10- CAC3/FC (9/1, v:v); S11- CAC4/FC (3/1, v:v); S12- CAC4/FC (9/1, v:v); S13-SC.

Para a carbonização da casca de arroz, utilizou-se um carbonizador (recipiente metálico de dez litros, com orifícios e com chaminé de dois metros), tendo em seu interior álcool e papel em combustão e sob o qual inseriu-se a casca de arroz, que sofreu carbonização, processo de combustão incompleta, em decorrência da baixa concentração de oxigênio. Para obtenção das diferentes granulometrias de CAC, foram utilizadas quatro peneiras com malhas: maior que $2 \mathrm{~mm}$; entre 1 e $2 \mathrm{~mm}$; de 0,5 a $1 \mathrm{~mm}$ e de 0,25 a $0,5 \mathrm{~mm}$. Os materiais foram misturados manualmente, juntamente com a adubação de base (40 $\mathrm{g} \mathrm{L}^{-1}$ de NPK 4-14-8; $10 \mathrm{~g} \mathrm{~L}^{-1} \mathrm{de}$ superfosfato simples [20\% de $\mathrm{P}_{2} \mathrm{O}_{5}$ e $14 \%$ de $\mathrm{SO}_{4}$ ] e $5 \mathrm{~g} \mathrm{~L}^{-}$ ${ }^{1}$ de FTE-BR12 [9\% Zn, 3\% Fe, 2\% Mn, 0,1\% Mo, 1,8\% B, $0,8 \% \mathrm{Cu}])$.

A caracterização física e química dos substratos foi realizada no laboratório de Solos da Embrapa Florestas, conforme a metodologia descrita na Instrução Normativa no 17 do Ministério da Agricultura, Pecuária e Abastecimento (Mapa, 2007), obtendo-se os valores de densidade aparente (Dap), capacidade de retenção de água a uma tensão de $10 \mathrm{~cm}$ (CRA 10, ou microporosidade), potencial hidrogeniônico $(\mathrm{pH})$ e condutividade elétrica (CE). A partir desses resultados, obtiveram-se a porosidade total $(\mathrm{Pt})$ e a macroporosidade (Macro). A Pt corresponde ao volume 
de água retido no substrato na tensão $0 \mathrm{hPa}$ (totalmente saturado), enquanto a macroporosidade consiste na diferença entre a Pt do substrato e o volume de água retido a $10 \mathrm{hPa}$ (CRA 10, ou microporosidade) (De boodt \& Verdonck, 1972).

Realizou-se semeadura direta de Eucalyptus camaldulensis em tubetes de $55 \mathrm{~cm}^{3}$. Para tanto, utilizouse um semeador manual e cada tubete recebeu em torno de quatro sementes, as quais foram cobertas com uma camada (em torno de $0,5 \mathrm{~cm}$ ) de vermiculita fina. Após a semeadura, as bandejas permaneceram por 60 dias em estufa de vidro $(6,65 \times$ x 7,90 m), modelo capela, com aberturas laterais e zenitais para saída de ar quente, recebendo, em média, três irrigações diárias de dez minutos, com vazão de 144 L/ hora. Posteriormente, foram transferidas para a área de rustificação (quatro irrigações diárias de 30 minutos, com vazão de $97 \mathrm{~L} /$ hora), onde foram expostas diretamente ao sol por 30 dias. Foi realizado o raleamento 20 dias após a semeadura, deixando-se como remanescente a muda mais central do tubete e com maior crescimento da parte aérea.

Aos 30 dias, aumentou-se o espaçamento entre as mudas, deixando o espaço de uma célula da bandeja, momento em que se iniciou a adubação de crescimento ( $4 \mathrm{~kg}$ de ureia, $3 \mathrm{~kg}$ de superfosfato simples, $0,25 \mathrm{~kg}$ de FTE BR 10 (7\% Zn, $4 \%$ Fe, $4 \%$ Mn, 0,1\% Mo, 2,5\% B, 0,8\% Cu) e 3 $\mathrm{kg}$ de cloreto de potássio por $\mathrm{m}^{3}$ de substrato), realizada a cada sete dias até os 60 dias, momento de transferência das mudas para a área de pleno sol, onde se realizou adubação de rustificação (4 kg de sulfato de amônio, $10 \mathrm{~kg}$ de superfosfato simples, $4 \mathrm{~kg}$ de cloreto de potássio, $1 \mathrm{~kg}$ de FTE BR 10 por $\mathrm{m}^{3}$ de substrato), realizada também a cada sete dias até os 90 dias.Para o preparo das adubações, os fertilizantes foram misturados com água e a solução resultante foi aplicada nas mudas com regador manual, aplicando-se aproximadamente $5 \mathrm{ml}$ por tubete.

Para a avaliação da qualidade das mudas, foi mensurada a altura da parte aérea (régua) e o diâmetro de colo (paquímetro digital), aos 90 dias, de todas as plantas. As análises destrutivas foram realizadas em cinco plantas por repetição, sendo elas: fitomassas secas das partes aérea e radicial ( 48 horas em estufa a $65^{\circ} \mathrm{C}$ ), pesadas em balança analítica de precisão $0,001 \mathrm{~g}$; facilidade de retirada do tubete e agregação das raízes ao substrato. Para as avaliações de facilidade de retirada das mudas do tubete e de agregação das raízes ao substrato, foi utilizada a metodologia descrita em Wendling et al. (2007). Este método consiste em atribuir notas de zero a dez às variáveis, sendo zero a dificuldade máxima e dez a facilidade máxima de retirada das mudas com as mãos, após três batidas na parte superior do tubete no canto da bandeja. Quanto à agregação das raízes ao substrato, as mudas sem os tubetes foram soltas em queda livre a um metro do solo, sendo atribuída ao torrão uma nota de zero a dez, sendo zero para a muda totalmente esboroada e dez para o torrão $100 \%$ íntegro. A fim de avaliar o equilíbrio de crescimento, foi calculado o índice morfológico que é a relação entre altura e diâmetro de colo (H/DC).

O delineamento experimental utilizado foi o inteiramente casualizado, com cinco repetições de 10 plantas e 13 tratamentos. Os dados referentes às características biométricas das mudas foram submetidos ao teste de Bartlett ( $\mathrm{p}<0,05)$, a fim de se verificar a condição de homogeneidade de variância e, em seguida, à análise de variância ( $p<0,01$ e p < 0,05), prosseguindo para o teste de Scott-Knott ( $p<0,01$ e $p<0,05)$, visando a observar as diferenças entre as médias. Para verificar a influência das propriedades físicas e químicas dos substratos nas variáveis biométricas, aplicou-se a análise de correlação de Pearson.

\section{RESULTADOS E DISCUSSÃO}

A análise de variância revelou efeito significativo do substrato para todas as variáveis analisadas (Tabela 1).

Para a altura das mudas, diâmetro do colo, fitomassas secas aérea e radicial, aos 90 dias, verificou-se superioridade do tratamento 50 CAC/ VM (1/1, v:v) em comparação com os demais substratos, por apresentar as maiores mudas com maiores fitomassas. Além desse, os substratos CAC3, CAC4, CAC2/FC (1/1, v:v), CAC4/FC (9/1, v:v), FC e SC apresentaram altura das mudas considerada apta para o plantio (entre 15 e $25 \mathrm{~cm}$ ), segundo Wendling \& Dutra (2010) (Tabela 2).

Pode-se verificar que a adição de FC aos componentes $\mathrm{CAC} 3$ e CAC4 acarretou pequeno decréscimo do crescimento das mudas (Tabela 2), constatando-se que nem sempre a combinação de dois componentes proporciona melhorias na formação da muda, pois sua eficácia depende muito dos materiais utilizados na mistura. Resultados similares foram encontrados por Kratz \& Wendling (2013), em cujos trabalhos a FC e a CAC pura não proporcionaram crescimento adequado às mudas de Eucalyptus dunnii, quando comparados com os efeitos de SC e CAC/ VM (1/1, v:v). Já, para E. grandis x E. urophylla, o substrato CAC/ VM (1/1, v:v) proporcionou menor crescimento, em comparação com aqueles obtidos com FC (Simões et al., 2012). A combinação dos componentes FC e CAC (1:1, v/v) proporcionou maior crescimento das mudas de Eucalyptus urophylla $\mathrm{x}$ E. grandis $(37,5 \mathrm{~cm} \mathrm{e}$ $3,56 \mathrm{~mm}$ ), quando comparado com os obtidos com a utilização destes componentes puros (Silva et al. 2012). Estes resultados indicam que não existe um substrato ideal para todas as espécies, mesmo em se tratando de espécies pertencentes ao mesmo gênero.

Da mesma maneira que a altura, o diâmetro de colo das plantas aos 90 dias apresentou para o substrato CAC/ 
VM (1/1, v:v) espessura maior que a obtida para os demais, seguindo-se as espessuras obtidas para SC e CAC3. Os demais tratamentos apresentaram diâmetros de colo inferiores ao mínimo indicado $(2 \mathrm{~mm})$ para o plantio em campo (Wendling \& Dutra, 2010) (Tabela 3), embora próximos aos observados em outras pesquisas com eucalipto. Kratz \& Wendling (2013), quando utilizaram o SC, observaram diâmetro de 1,79 mm em mudas de E. dunnii, enquanto aquelas produzidas em substratos à base de CAC em diferentes granulometrias e FC apresentaram diâmetro médio de 1,10 mm; Trigueiro \& Guerrini (2003) obtiveram diâmetros de colo de 1,85 mm em mudas de E. grandis, produzidas em SC, e Freitas et al. (2005), de 2,0 mm, em mudas de E.grandis e 1,80 mm em E. saligna, produzidas em CAC/casca de eucalipto (1/1, v:v).

No que se refere à relação H/DC, que variou de 9,41 a 12,11 , os resultados obtidos para todos os substratos ficaram fora da faixa considerada adequada $(5,4$ a 8,1$)$ por Carneiro (1995) (Tabela 2). Logo, verifica-se que esta faixa re- comendada não é a mais indicada para o Eucalipto, conforme já observado em outros trabalhos, em que a relação H/ DC sempre aparece como superior a oito. Em trabalho com mudas de E. grandis, Trigueiro \& Guerrini (2003) verificaram H/DC variando de 10,74 a 13,90; Simões et al. (2012), para E. grandis x E. urophylla, entre 9,39 e 12,13; e Kratz \& Wendling (2013), em E. dunnii, de 6,52 a 8,95. Cabe observar que o substrato que proporcionou o maior crescimento em altura e diâmetro, CAC/ VM (1/1, v:v), apresentou índice H/DC de 11,24, valor próximo ao observado em substratos com baixo crescimento. Desta forma, a referida relação não pode ser utilizada como um fator único para avaliação de qualidade de mudas. Segundo Gomes et al. (2002), a relação H/DC não é um índice de grande importância, conforme citado em vários trabalhos, visto que apresentou contribuição relativa de apenas $0,66 \%$ para a avaliação da qualidade de mudas de E. grandis.

A quantificação da fitomassa para a avaliação de qualidade de mudas, por outro lado, é de grande importância,

Tabela 1: Análise de variância para altura (H), diâmetro de colo (DC), relação altura e diâmetro de colo (H/DC), fitomassa seca aérea (FSA), fitomassa seca radicial (FSR), relação fitomassa seca aérea e fitomassa seca radicial (FSA/FSR), facilidade de retirada do tubete (FRT) e agregação das raízes ao substrato (AG) de mudas de E. camaldulensis, produzidas em diferentes substratos, aos 90 dias.

\begin{tabular}{lcccccccc}
\hline $\begin{array}{l}\text { Causa da } \\
\text { Variação }\end{array}$ & \multirow{2}{*}{ GL } & H & DC & H/DC & FSA & FSR & FRT & AG \\
\hline Substrato & 12 & $50,070^{* *}$ & $0,354^{* *}$ & $7,522^{* *}$ & $0,110^{* *}$ & $0,001^{* *}$ & $1,326^{* *}$ & $6,028^{* *}$ \\
Resíduo & 56 & 2,977 & 0,019 & 1,620 & 0,002 & $3 \times 10^{-4}$ & 0,588 & 0,692 \\
Média & - & 16,09 & 1,48 & 10,89 & 0,289 & 0,025 & 9,00 & 9,00 \\
\hline CV $_{\text {exp. }}(\%)$ & - & 10,72 & 9,27 & 11,69 & 17,48 & 20,78 & 8,11 & 9,25 \\
\hline
\end{tabular}

** - significativo a $1 \%$ de probabilidade pelo teste F. GL - graus de liberdade, $\mathrm{CV}_{\text {exp. }}$ - coeficiente de variação experimental.

Tabela 2: Altura (H), Diâmetro de Colo (DC), relação altura e diâmetro de colo (H/DC), fitomassa seca aérea (FSA), fitomassa seca radicial (FSR), facilidade de retirada do tubete (FRT) e agregação das raízes ao substrato (AG) de mudas de E. camaldulensis, produzidas em diferentes substratos, aos 90 dias.

\begin{tabular}{|c|c|c|c|c|c|c|c|}
\hline \multirow{2}{*}{ Substrato } & \multirow{2}{*}{$\begin{array}{c}\mathbf{H} \\
(\mathrm{cm})\end{array}$} & \multirow{2}{*}{$\frac{\mathrm{DC}}{(\mathrm{mm})}$} & \multirow{2}{*}{ H/DC } & FSA & FSR & \multirow{2}{*}{ FRT } & \multirow{2}{*}{ AG } \\
\hline & & & & \multicolumn{2}{|c|}{$\left(\mathrm{mg}^{-1}\right)$} & & \\
\hline$\overline{\mathrm{CAC}}$ & $14,18 \mathrm{c}$ & $1,49 \mathrm{c}$ & $9,48 \mathrm{c}$ & $0,250 \mathrm{c}$ & $0,137 \mathrm{c}$ & $10,00 \mathrm{a}$ & $8,00 \mathrm{~b}$ \\
\hline CAC1 & $14,20 \mathrm{c}$ & $1,28 \mathrm{~d}$ & $11,05 \mathrm{c}$ & $0,181 \mathrm{c}$ & $0,096 \mathrm{c}$ & $10,00 \mathrm{a}$ & $8,00 \mathrm{~b}$ \\
\hline CAC2 & $13,90 \mathrm{c}$ & $1,39 \mathrm{~d}$ & $9,93 \mathrm{c}$ & $0,211 \mathrm{c}$ & $0,114 \mathrm{c}$ & $10,00 \mathrm{a}$ & $9,00 \mathrm{a}$ \\
\hline CAC3 & $17,73 \mathrm{~b}$ & $1,65 \mathrm{~b}$ & $10,73 \mathrm{c}$ & $0,305 \mathrm{~b}$ & $0,141 \mathrm{c}$ & $9,00 \mathrm{a}$ & $9,00 \mathrm{a}$ \\
\hline CAC4 & $18,43 \mathrm{~b}$ & $1,31 \mathrm{~d}$ & $14,04 \mathrm{a}$ & $0,258 \mathrm{c}$ & $0,101 \mathrm{c}$ & $10,00 \mathrm{a}$ & $10,00 \mathrm{a}$ \\
\hline $\mathrm{FC}$ & $16,53 \mathrm{~b}$ & $1,56 \mathrm{c}$ & $10,54 \mathrm{c}$ & $0,296 \mathrm{~b}$ & $0,166 \mathrm{~b}$ & $10,00 \mathrm{a}$ & $9,00 \mathrm{a}$ \\
\hline CAC/ VM (1/1, v:v) & $25,03 \mathrm{a}$ & $2,22 \mathrm{a}$ & $11,24 \mathrm{c}$ & $0,757 \mathrm{a}$ & $0,398 \mathrm{a}$ & $10,00 \mathrm{a}$ & $10,00 \mathrm{a}$ \\
\hline CAC2/ FC (1/1, v:v) & $15,73 \mathrm{~b}$ & $1,29 \mathrm{~d}$ & $12,11 \mathrm{~b}$ & $0,233 \mathrm{c}$ & $0,116 \mathrm{c}$ & $8,00 \mathrm{~b}$ & $8,00 \mathrm{~b}$ \\
\hline CAC3/ FC (1/1, v:v) & $12,66 \mathrm{c}$ & $1,34 \mathrm{~d}$ & $9,41 \mathrm{c}$ & $0,222 \mathrm{c}$ & $0,112 \mathrm{c}$ & $8,00 \mathrm{~b}$ & $7,00 \mathrm{~b}$ \\
\hline CAC3/ FC (9/1, v:v) & $14,33 \mathrm{c}$ & $1,35 \mathrm{~d}$ & $10,60 \mathrm{c}$ & $0,227 \mathrm{c}$ & $0,106 \mathrm{c}$ & $9,00 \mathrm{a}$ & $8,00 \mathrm{~b}$ \\
\hline CAC4/FC (3/1, v:v) & $13,93 \mathrm{c}$ & $1,22 \mathrm{~d}$ & $11,39 \mathrm{c}$ & $0,188 \mathrm{c}$ & $0,082 \mathrm{c}$ & $10,00 \mathrm{a}$ & $9,00 \mathrm{a}$ \\
\hline CAC4/ FC (9/1, v:v) & $15,96 \mathrm{~b}$ & $1,48 \mathrm{c}$ & $10,74 \mathrm{c}$ & $0,277 \mathrm{~b}$ & $0,116 \mathrm{c}$ & $10,00 \mathrm{a}$ & $9,00 \mathrm{a}$ \\
\hline $\mathrm{SC}$ & $16,53 \mathrm{~b}$ & $1,70 \mathrm{~b}$ & $9,69 \mathrm{c}$ & $0,357 \mathrm{~b}$ & $0,201 \mathrm{~b}$ & $10,00 \mathrm{a}$ & $9,00 \mathrm{a}$ \\
\hline
\end{tabular}

Médias seguidas pela mesma letra na coluna não diferem entre si pelo teste de Scott-Knott a $1 \%$ de probabilidade. CAC - Casca de arroz carbonizada original; CAC1 - Casca de arroz carbonizada com granulometria maior que 2 mm; CAC2 - Casca de arroz carbonizada com granulometria entre 1 - $2 \mathrm{~mm}$; CAC3 - Casca de arroz carbonizada com granulometria entre 0,5 - $1 \mathrm{~mm}$; CAC4 - Casca de arroz carbonizada com granulometria menor que $0,5 \mathrm{~mm}$; FC - Fibra de Coco; VM - Vermiculita; SC - Substrato comercial à base de casca de pinus e vermiculita. 
haja vista que as mudas com maior valor apresentam maior resistência às condições adversas do campo, promovendo maior sobrevivência e evitando gastos com replantio (Gomes et al., 2002).

As fitomassas secas aérea e radicial apresentaram comportamento semelhante ao das demais variáveis biométricas e o substrato CAC/ VM (1/1, v:v) proporcionou a maior produção de massa seca (Tabela 2), resultados similares aos observados por Kratz \& Wendling (2013) em mudas de E. dunnii, para as quais o substrato CAC/ VM $(1 / 1, v: v)$, seguido do SC, proporcionou maior acúmulo de fitomassa, quando comparado com os substratos à base de diferentes granulometrias de CAC e FC. Entretanto, Silva et al. (2012) e Simões et al. (2012) verificaram que a utilização dos componentes FC e CAC, puros ou combinados, apresentaram crescimento satisfatório de mudas de Eucalyptus urophylla x E. grandis e de Eucalyptus grandis x Eucalyptus urophylla, respectivamente, denotando a necessidade de se avaliar os substratos antes de utilizá-lo em escala, na produção comercial de mudas.

Todos os tratamentos apresentaram altos índices de facilidade de retirada das mudas do tubete (acima de 8), da mesma forma que para a agregação das raízes ao substrato; apenas o substrato CAC3/ FC (1/1, v:v) apresentou agregação média $(7,0)$ (Tabela 2$)$. Estes resultados geram agilidade aos processo de expedição e de plantio das mudas em campo.

O substrato para produzir mudas em tubetes deve ser agregado o suficiente para que o torrão em volta da muda não se rompa, quando a embalagem for retirada para plantio ou transporte, ocasionando exposição das raízes ao ressecamento e dificultando a pega e a sobrevivência das mudas (Wendling \& Delgado, 2008). No entanto, se o substrato for muito coeso, haverá dificuldades em sua retirada da embalagem, podendo-se romper as raízes ou provocar danos ao crescimento radicial das mudas (Trigueiro \& Guerrini, 2003).

A agregação das raízes ao substrato está diretamente relacionada com a fitomassa seca aérea $\left(\mathrm{R}=0,77^{* *}\right)$, com a altura $\left(\mathrm{R}=0,94^{* *}\right)$ e com o diâmetro de colo $\left(\mathrm{R}=0,78^{* *}\right)$ (Tabela 3), denotando que as mudas com maiores crescimentos aéreo e radicular apresentam maior agregação do sistema radicial. Esses resultados estão de acordo com os obtidos por Trigueiro e Guerrini (2003), com mudas de E.grandis; os de Kratz \& Wendling (2013), com E. dunnii, e os de Kratz et al. (2013a), com E. benthamii e Mimosa scabrella.

Pode-se observar que as variáveis estudas não se correlacionaram apenas com a FRT, sendo que as demais variáveis apresentaram correlação positiva entre si, denotando que as plantas apresentaram crescimento equilibrado (Tabela 3).

A densidade aparente, o $\mathrm{pH}$ e a CTC apresentaram influência significativa no crescimento das mudas (Tabela 4), denotando, de maneira geral, que os substratos com maior densidade, menor $\mathrm{pH}$ e maior CTC, proporcionaram maior crescimento (Tabelas 2 e 5). No entanto, para a densidade, esta afirmação não é totalmente aplicada, visto que o crescimento das mudas produzidas com SC $\left(0,49 \mathrm{~g} / \mathrm{cm}^{3}\right)$ foi menor do que com CAC/VM $(0,36$ $\left.\mathrm{g} / \mathrm{cm}^{3}\right)$, assim como para o $\mathrm{pH}$, haja vista que o CAC/ VM (1/1, v:v) apresentou pH de 8,12, proporcionando crescimento adequado das mudas, seguido do CAC4, com 8,44.

Em se tratando das propriedades físicas, verificouse que o aumento do tamanho das partículas de CAC proporcionou redução da densidade aparente e da microporosidade e acréscimo proporcional da porosidade total e da macroporosidade. Ao mesmo tempo, a adição de FC nas diferentes granulometrias de CAC proporcionou diferentes respostas, dependendo do tamanho da partícula, podendo diminuir ou aumentar a densidade e a porosidade (Tabela 5). Entretanto, as diferenças observadas nas propriedades físicas, exceto a densidade, não apresentaram correlação com o crescimento das mudas de E. camaldulensis (Tabela 4), mostrando que a combinação dos fatores é mais importante do que os fatores individuais na produção de mudas (Kratz et al., 2012).

Quanto ao $\mathrm{pH}$, diferentemente de outras espécies, o Eucalyptus tem mostrado grande plasticidade, denotando ser menos exigente quanto a esta variável, ou seja, mais tolerante às variações desse índice. Isto pode ser observado com mudas de E. benthamii, produzidas em

Tabela 3: Correlações entre as variáveis altura (H), diâmetro do colo (DC), fitomassa seca aérea (FSA), fitomassa seca radicial (FSR), facilidade de retirada das mudas do tubete (FRT) e agregação das raízes ao substrato (AG), aos 90 dias, em mudas de E. camaldulensis

\begin{tabular}{lcccccc}
\hline & H & DC & FSA & FSR & FRT & AG \\
\hline H & $1,00^{* *}$ & & & & & \\
DC & $0,84^{* * *}$ & $1,00^{* *}$ & & & & \\
FSA & $0,92^{* *}$ & $0,95^{* *}$ & $1,00^{* *}$ & & & \\
FSR & $0,86^{* *}$ & $0,96^{* *}$ & $0,98^{* *}$ & $1,00^{* *}$ & & \\
FRT & 0,29 ns & $0,32^{\text {ns }}$ & $0,27^{\text {ns }}$ & $0,26^{\text {ns }}$ & $1,00^{* *}$ & $1,00^{* *}$ \\
AG & $0,94^{* *}$ & $0,78^{* *}$ & $0,84^{* *}$ & $0,77^{* *}$ & $0,51^{*}$ & 1.4 \\
\hline
\end{tabular}

**, ${ }^{*}$ - significativo a 1 e $5 \%$ de probabilidade, respectivamente; ${ }^{\text {ns }}$ - não significativo, pelo teste T.

Rev. Ceres, Viçosa, v. 63, n.3, p. 348-354, mai/jun, 2016 
substratos com distintos valores de $\mathrm{pH}(5,5$ a 9,1$)$, obtendo-se crescimento adequado em todos os substratos (Kratz et al., 2013b). Segundo Valeri \& Corradini (2000), em substratos com $\mathrm{pH}$ abaixo de 5,0, podem ocorrer as deficiências de nitrogênio, potássio, cálcio, magnésio e boro; enquanto em pH acima de 6,5, são esperadas deficiências de fósforo, ferro, manganês, zinco e cobre. Entretanto, neste estudo, não foram observados sintomas de deficiência ou toxidez.

Todos os substratos com apenas CAC apresentaram baixa CTC (abaixo de $100 \mathrm{mmol} / \mathrm{dm}^{3}$ ), enquanto aqueles com a presença de FC, de SC e de VM apresentaram CTC adequada (acima de $200 \mathrm{mmol} / \mathrm{dm}^{3}$ ), segundo a recomendação de Gonçalves e Poggiani (1996) (Tabela 5). Conforme citado por Kämpf (2005), os problemas de substratos com falta ou excesso de retenção de nutrientes podem ser contornados, em parte, pelo uso de misturas com componentes que apresentem maior valor de CTC, conforme observado nos substratos com FC e vermiculita (Tabela 4). Kratz \& Wendling (2013) obtiveram resultados similares, em que o substrato CAC/VM proporcionou um bom crescimento das mudas, em relação àqueles à base de CAC e FC.

Os resultados obtidos neste estudo não inviabilizam a utilização dos substratos à base de CAC em diferentes granulometrias, $\mathrm{FC}$ e comercial à base de casca de pinus semi- decomposta. Apesar de apresentarem crescimentos menores do que os obtidos com o substrato CAC/ $\mathrm{VM}$, as mudas produzidas nos substratos CAC3, CAC4, CAC2/ FC (1/1, v:v), CAC4/ FC (9/1, v:v), FC e SC apresentaram aptidão morfológica para o plantio, segundo Wendling \& Dutra (2010) (Tabela 2). Além disso, os referidos substratos apresentaram boas características físicas e químicas, devendo, no entanto, haver ajustes na adubação para que possam proporcionar melhores crescimentos.

Tabela 4: Correlações entre as propriedades físicas e químicas dos substratos e variáveis biométricas das mudas de E. camaldulensis: altura (H), diâmetro do colo (DC), fitomassa seca aérea (FSA), fitomassa seca radicial (FSR), facilidade de retirada do tubete (FRT), agregação das raízes ao substrato (AG), densidade aparente (Dap), porosidade total (Pt), macroporosidade (Macro), microporosidade (Micro), potencial hidrogeniônico $(\mathrm{pH})$, condutividade elétrica (CE) e capacidade de troca catiônica (CTC)

\begin{tabular}{llllllll}
\hline & Dap & Pt & Macro & Micro & p H & CE & CTC \\
\hline H & $0,56^{*}$ & $-0,21^{\text {ns }}$ & $-0,10^{\text {ns }}$ & $-0,03^{\text {ns }}$ & $-0,43^{*}$ & $0,16^{\text {ns }}$ & $0,28^{\text {ns }}$ \\
DC & $0,64^{* *}$ & $-0,02^{\text {ns }}$ & $0,17^{\text {ns }}$ & $-0,13^{\text {ns }}$ & $-0,50^{*}$ & $0,01^{\text {ns }}$ & $0,40^{*}$ \\
FSA & $0,58^{*}$ & $-0,07^{\text {ns }}$ & $0,07^{\text {ns }}$ & $-0,09^{\text {ns }}$ & $-0,60^{* *}$ & $-0,06^{\text {ns }}$ & $0,42^{*}$ \\
FSR & $0,57^{*}$ & $-0,05^{\text {ns }}$ & $0,19^{\text {ns }}$ & $-0,18^{\text {ns }}$ & $-0,62^{* *}$ & $-0,06^{\text {ns }}$ & $0,47^{*}$ \\
FRT & $0,48^{*}$ & $0,31^{\text {ns }}$ & $0,17^{\text {ns }}$ & $0,07^{\text {ns }}$ & $0,15^{\text {ns }}$ & $0,08^{\text {ns }}$ & $-0,18^{\text {ns }}$ \\
AG & $0,57^{*}$ & $-0,01^{\text {ns }}$ & $-0,11^{\text {ns }}$ & $0,08^{\text {ns }}$ & $-0,24^{\text {ns }}$ & $0,16^{\text {ns }}$ & $0,06^{\text {ns }}$ \\
\hline
\end{tabular}

**, * - significativo a 1 e $5 \%$ de probabilidade, respectivamente; ns - não significativo, pelo teste T.

Tabela 5: Densidade aparente (Dap), porosidade total (Pt), macroporosidade (Macro), microporosidade (Micro), potencial hidrogeniônico $(\mathrm{pH})$, condutividade elétrica $(\mathrm{CE})$ e capacidade de troca catiônica $(\mathrm{CTC})$ para diferentes substratos formados à base de CAC.

\begin{tabular}{|c|c|c|c|c|c|c|c|}
\hline \multirow{2}{*}{ Substrato } & Dap & $\mathbf{P t}$ & Macro & Micro & \multirow{2}{*}{$\frac{\mathrm{pH}}{\left(\mathrm{H}_{2} \mathrm{O}\right)}$} & \multirow{2}{*}{ 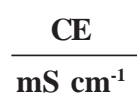 } & \multirow{2}{*}{$\frac{\text { CTC }}{\left(\mathrm{mmol}_{\mathrm{c}} \mathbf{d m}^{-3}\right)}$} \\
\hline & $\left(\mathrm{g} / \mathrm{cm}^{3}\right)$ & & $(\%)$ & & & & \\
\hline $\mathrm{CAC}$ & 0,29 & 72,03 & 27,74 & 44,29 & 8,58 & 1,48 & 37,6 \\
\hline CAC1 & 0,19 & 53,08 & 30,25 & 22,83 & 8,51 & 1,26 & 85,6 \\
\hline CAC2 & 0,26 & 63,17 & 32,08 & 31,10 & 8,47 & 1,30 & 68,8 \\
\hline CAC3 & 0,33 & 54,33 & 11,27 & 43,06 & 8,47 & 1,68 & 79,6 \\
\hline CAC4 & 0,32 & 51,98 & 5,32 & 46,66 & 8,44 & 1,94 & 79,8 \\
\hline FC & 0,41 & 58,67 & 21,61 & 37,05 & 5,81 & 0,74 & 257,6 \\
\hline CAC/ VM $(1 / 1, \mathrm{v}: \mathrm{v})$ & 0,36 & 60,32 & 16,57 & 43,75 & 8,12 & 1,10 & 127,2 \\
\hline $\mathrm{CAC} 2 / \mathrm{FC}(1 / 1, \mathrm{v}: \mathrm{v})$ & 0,27 & 53,51 & 7,62 & 45,88 & 6,63 & 0,71 & 259,2 \\
\hline CAC3/ FC (1/1, v:v) & 0,24 & 54,85 & 14,42 & 40,44 & 6,99 & 0,46 & 210,0 \\
\hline CAC3/ FC (9/1, v:v) & 0,23 & 60,38 & 14,48 & 45,89 & 7,72 & 0,29 & 116,2 \\
\hline CAC4/FC (3/1, v:v) & 0,34 & 62,79 & 7,15 & 55,63 & 7,34 & 0,41 & 125,2 \\
\hline CAC4/FC (9/1, v:v) & 0,38 & 61,73 & 5,6 & 56,13 & 7,78 & 0,25 & 103,0 \\
\hline $\mathrm{SC}$ & 0,49 & 49,31 & 14,38 & 42,93 & 6,06 & 0,76 & 438,4 \\
\hline
\end{tabular}

CAC - Casca de arroz carbonizada; CAC1 - Casca de arroz carbonizada com granulometria maior que 2 mm; CAC2 - Casca de arroz carbonizada com granulometria entre 1 - $2 \mathrm{~mm}$; CAC3 - Casca de arroz carbonizada com granulometria entre 0,5 - 1 mm; CAC4 - Casca de arroz carbonizada com granulometria menor que $0,5 \mathrm{~mm}$; FC - Fibra de Coco; VM - Vermiculita; SC - Substrato comercial à base de casca de pinus e vermiculita. 


\section{CONCLUSÕES}

Os substratos formados por CAC, em diferentes granulometrias e em mistura com os outros componentes, são viáveis tecnicamente para a produção de mudas de Eucalyptus camaldulensis, embora aquele formado pela mistura de $50 \%$ de CAC e VM tenha resultado em maior crescimento das mudas. Aliado a isto, a densidade aparente, o pH e a capacidade de troca catiônica dos substratos são importantes na produção de mudas, pois apresentam correlações significativas com variáveis biométricas.

\section{REFERENCIAS}

Caldeira MVW, Wendling I, Penchel RM, Gonçalves EO, Kratz D \& Trazzi PA (2011) Propriedades de substratos para produção de mudas florestais. In: Caldeira MVW, Garcia GO, Gonçalves EO, Arantes MDC \& Fiedler NC (Eds.) Contexto e Perspectivas da Área Florestal no Brasil. Alegre, Suprema. p.141-160.

Carneiro JGA (1995) Produção e controle de qualidade de mudas florestais. Curitiba, UFPR/FUPEF. 451p.

Couto M, Wagner Junior A \& Quezada AC (2003) Efeito de diferentes substratos durante a aclimatização de plantas micropropagadas do porta-enxerto mirabolano $29 \mathrm{c}$ (Prunuscerasiferaehrh.) em casa de vegetação. Revista Brasileira de Agrociência, 9:125-128.

De Boodt M \& Verdonck O (1972) The physical properties of the substrates. Acta Horticulturae, 26:37-44.

Fermino MH (2003) Métodos de análise para caracterização de física de substratos. Tese de Doutorado. Universidade Federal do Rio Grande do Sul, Porto Alegre. 89p.

Freitas TAS, Barroso DG, Carneiro JGA, Penchel RM, Lamônica KR \& Ferreira DA (2005) Desempenho radicular de mudas de eucalipto produzidas em diferentes recipientes e substratos. Revista Árvore, 29:853-861.

Gomes JM, Couto L, Leite HG, Xavier A \& Garcia SLR (2002) Parâmetros morfológicos na avaliação da qualidade de mudas de Eucalyptus grandis. Revista Árvore, 26:655-664.

Gonçalves JLM, Santarelli ED, Moraes Neto SP \& Manara MP (2000) Produção de mudas de espécies nativas: substrato, nutrição, sombreamento e fertilização. In: Gonçalves JLM \& Benedetti V (Eds.) Nutrição e fertilização florestal. Piracicaba, IPEF. p. 309-350.

Gonçalves LM \& Poggiani F (1996) Substratos para produção de mudas florestais. In: $13^{\circ}$ Congresso Latino Americano de Ciência do Solo, Águas de Lindóia. Anais, Sociedade Latino Americana de Ciência do Solo. CD-ROM.

Hartmann HT, Kester DE, Davies Jr. FT \& Geneve R (2011) Plant propagation: principles and practices. $8^{\mathrm{a}}$ ed. Boston, Prentice-Hall. 915p.

IBA - Industria Brasileira de Árvores (2014) IBÁ 2014 ano base 2013. Brasília, IBÁ. 100p.

Kämpf AN (2005) Substrato. In: Kämpf NA (Ed.) Produção comercial de plantas ornamentais. $2^{\mathrm{a}}$ ed. Guaíba, Agrolivros, p.4572.

Kratz D \& Wendling I (2013) Produção de mudas de Eucalyptus dunnii em substratos renováveis. Floresta, 43:125-136.

Kratz D, Wendling I \& Pires PP (2012) Miniestaquia de Eucalyptus benthamii x E. dunnii em substratos à base de casca de arroz carbonizada. Scientia Forestalis, 40:547-556.
Kratz D, Wendling I, Nogueira AC \& Souza PVD (2013a) Substratos renováveis na produção de mudas de Eucalyptus benthamii. Ciência Florestal, 23:607-621.

Kratz D, Wendling I, Nogueira AC \& Souza PVD (2013b) Utilização de resíduos urbanos e agroflorestais para produção de mudas de Eucalyptus benthamii e Mimosa scabrella. Floresta e Ambiente, 20:530-537.

Melo LA, Pereira GA, Moreira EJC, Davide AC, Silva EV \& Teixeira LAF (2014) Crescimento de mudas de Eucalyptus grandis e Eremanthus erythropappus sob diferentes formulações de substrato. Floresta e Ambiente, 21:234-242.

MAPA - Ministério da Agricultura, Pecuária e Abastecimento (2007) Instrução Normativa SDA No 17 , de 24 de maio de 2007. Métodos analíticos oficiais para análise de substratos para plantas e condicionadores de solo. DOU, 24/05/2007, Seção 1, p.9.

MAPA - Ministério da Agricultura, Pecuária e Abastecimento (2015) Resolução DSV/SDA Nº 6, de 6 de outubro de 2015. Estabelece a coleta de amostras para análise das partidas importadas de turfa de Sphagnum provenientes de qualquer origem autorizada. DOU, 07/10/2015, Seção 1, p.7.

Shimizu JY, Klein H \& Oliveira JRV (2007) Diagnóstico das plantações florestais em Mato Grosso. Cuiabá, Central de Texto. $63 \mathrm{p}$.

Silva RBG, Simões D \& Silva M (2012) Qualidade de mudas clonais de Eucalyptus urophylla x E. grandis em função do substrato. Revista Brasileira Engenharia Agrícola e Ambiental, 16:297302.

Simões D, Silva RBG \& Silva MR (2012) Composição do substrato sobre o desenvolvimento, qualidade e custo de produção de mudas de Eucalyptus grandis Hill ex Maiden $\times$ Eucalyptus urophylla S. T. Blake. Ciência Florestal, 22:091-100.

Trigueiro RM \& Guerrini IA (2003) Uso de biossólido como substrato para produção de mudas de eucalipto. ScientiaForestalis, 64:150-162.

Valeri SV \& Corradini L (2000) Fertilização em viveiros para produção de mudas de Eucalyptus e Pinus. In: Gonçalves JLM \& Benedetti V (Ed.) Nutrição e fertilização florestal. Piracicaba, IPEF. p.167-189.

Wendling I \& Delgado ME (2008) Produção de mudas de araucária em tubetes. Colombo, Embrapa Florestas. 8p.

Wendling I \& Dutra LF (2010) Produção de mudas de eucalipto por sementes. In: Wendling I \& Dutra LF (Eds.) Produção de mudas de eucalipto. Colombo, Embrapa Florestas. p.13-47.

Wendling I, Guastala D \& Dedecek R (2007) Características físicas e químicas de substratos para produção de mudas de Ilex paraguariensis St.-Hil. Revista Árvore, 31:209-220. 